\title{
VISIONES DEFORMADAS DE LA CIENCIA TRANSMITIDAS POR LA ENSEÑANZA
}

\author{
FERNÁNDEZ, ISABEL ${ }^{1}$, GIL, DANIEL ${ }^{1}$, CARRASCOSA, JAIME ${ }^{1}$, CACHAPUZ, ANTÓNIO ${ }^{2}$ \\ Y PRAIA, JO $\tilde{A O}^{3}$ \\ 1 Universitat de València \\ 2 Universidade de Aveiro \\ ${ }^{3}$ Universidade de Porto
}

\begin{abstract}
Resumen. Numerosas investigaciones han mostrado que la enseñanza de las ciencias, incluso en el nivel universitario, apenas proporciona ocasión a los estudiantes de familiarizarse con las estrategias características del trabajo científico. Como consecuencia de ello las concepciones de los estudiantes, e incluso de los mismos profesores, acerca de la naturaleza de la ciencia no difieren de la visiones ingenuas adquiridas por impregnación social.

En este trabajo se argumenta la importancia de estas visiones deformadas como uno de los principales obstáculos para la renovación de la enseñanza de las ciencias y se analiza la atención concedida por la investigación didáctica al conjunto de deformaciones y reduccionismos.
\end{abstract}

Palabras clave. Naturaleza de la ciencia, visiones deformadas de la ciencia, concepciones docentes, formación del profesorado.

Summary. Many studies have shown that science education, even at university level, rarely gives students the opportunity to practise the strategies characteristic to the scientific approach. This results in the fact that students' and even science teachers' conceptions about the nature of science don't differ from so-called «folk» or «naïve» views, acquired by social impregnation. In this paper we discuss the importance of these distorted views as one of the main obstacles to renewal movements in science education and we analyse the attention paid by science education research to the different distortions and reductionisms.

Keywords. Nature of science, distorted views of science, teachers' conceptions, teachers' training.

\section{IMPORTANCIA DE LA CONCEPCIONES DOCENTES ACERCA DE LA CIENCIA}

La atención que la investigación en didáctica de las ciencias ha prestado al estudio de las preconcepciones de los profesores fue escasa hasta la segunda mitad de los años ochenta. Así, en 1987, Hewson y Hewson publicaron un interesante trabajo ( $\ll$ Science Teachers' conception of teaching: implications for teachers education») cuya idea central puede resumirse así: del mismo modo que los alumnos poseen preconcepciones, ideas y comportamientos intuitivos, que interfieren en la adquisición de los conocimientos científicos, cabe suponer también que los profesores poseemos preconcepciones acerca de la enseñanza que pueden entrar en conflicto con lo que la investigación ha mostrado acerca de la enseñanza y el aprendizaje de las ciencias.
Simultáneamente, se publica un trabajo de Gené y Gil (1987) en el que afirman: «Un primer error en el diseño de la formación inicial del profesorado estriba en concebir ésta como realmente inicial ignorando que los futuros profesores poseen ya unos conocimientos, plantean unos procedimientos y tienen unas actitudes hacia la enseñanza y el aprendizaje, que son el resultado de una formación adquirida "ambientalmente" a lo largo de los muchos años en que han seguido como alumnos las actuaciones de sus profesores. Se trata de una formación que tiene un gran peso por su carácter reiterado y al no estar sometida a una crítica explícita, aparece como "natural" sin que llegue a ser cuestionada efectivamente. Ignorar esta formación tiene los mismos efectos negativos que no 
tomar en consideración las preconcepciones de los alumnos a la hora de diseñar un determinado aprendizaje.»

Esta atención hacia las concepciones docentes se vio impulsada por la constatación de las diferencias existentes entre las aportaciones de la investigación en didáctica de las ciencias, incorporadas por los diseñadores de currículos, y lo que los profesores llevamos realmente a la práctica (Cronin-Jones, 1991; Barrow, 1991). Los resultados de estos estudios sobre la influencia que los docentes ejercemos en el proceso de implementación de los currículos mostraron la necesidad de cuestionar y modificar las «teorías implícitas» del profesorado acerca de cómo aprenden los alumnos y de cuál es la naturaleza del conocimiento científico, para que las propuestas fundamentadas de innovación de la enseñanza puedan ser llevadas correctamente a la práctica.

Comienza a producirse así un consenso creciente en torno a la necesidad de modificar la epistemología «espontánea» del profesorado, dado que la misma puede constituir un obstáculo capaz de bloquear los intentos de renovación de la enseñanza de las ciencias (Bell y Pearson, 1992; Gil, 1993; Désautels y Larochelle, 1998a; Meichtry, 1999...). En este sentido, Bell y Pearson (1992) han expresado con rotundidad la importancia de la epistemología de los docentes para la renovación de la enseñanza de las ciencias: «Empieza a comprenderse que, si se quiere cambiar lo que los profesores y los alumnos hacemos en las clases de ciencias, es preciso previamente modificar la epistemología de los profesores.»

De este modo, la investigación en torno a las concepciones docentes acerca de la ciencia y su enseñanza pasa a convertirse, desde finales de los años ochenta, en una potente línea de investigación de la que son exponente, además de un número creciente de artículos, diversas tesis doctorales (Porlán, 1989; Praia, 1995; Paixão, 1998; Fernández, 2000) o trabajos de recopilación como el volumen editado por McComas (1998) con el significativo título de The nature of science in science education.

Cabe recordar, por otra parte, que esta atención a las concepciones docentes sobre la naturaleza de la ciencia se había iniciado décadas atrás, aunque limitada a señalar las serias discrepancias entre la visión de la ciencia proporcionada por la epistemología contemporánea y ciertas concepciones docentes, ampliamente extendidas, marcadas por un empirismo e inductivismo extremo (Giordan, 1978; Gil, 1983; Hodson, 1985; Nussbaum, 1989). Este empiroinductivismo ha seguido siendo denunciado a lo largo de las dos últimas décadas (Cleminson, 1990; Meitchtry, 1999...).

Conviene señalar que esta idea, que atribuye la esencia de la actividad científica a la experimentación, coincide con la de «descubrimiento» científico propagada por los medios de comunicación, prensa, revistas, etc. (Lakin y Wellington, 1994).

Esta coincidencia de la imagen empirista que los docentes solemos tener sobre la ciencia y la que transmiten los media, en general, y la publicidad, en particular (Campario, Moya y Otero, 2001), permite conjeturar que las visiones de los profesores -o las que proporcionan los libros de texto (Selley, 1989; Stinner, 1992)- no serán, en general, muy diferentes de lo que podríamos denominar la imagen folk, «popular», socialmente aceptada de la ciencia. Por otra parte, dado que la enseñanza científica -incluida la universitaria- se ha reducido básicamente a la presentación de conocimientos ya elaborados, sin dar ocasión a los estudiantes de asomarse a las actividades características de la actividad científica, cabe esperar que esa imagen popular de la ciencia, en la que abundan los tópicos deformantes (como la imagen de los científicos asociada a «sabios despistados» que trabajan en solitario, aislados del mundo), persista incluso entre los profesores, influenciando negativamente nuestra enseñanza. De ahí la importancia de un estudio centrado en detectar la presencia y la extensión de las visiones deformadas de la ciencia que puedan constituir un obstáculo para la necesaria renovación de su enseñanza.

\section{VISIONES DEFORMADAS DE LA ACTIVI- DAD CIENTÍFICA}

Como acabamos de señalar, una de las razones que explican el interés por el estudio de las concepciones docentes sobre la naturaleza de la ciencia estriba en el convencimiento de que dichas concepciones incluyen reduccionismos y deformaciones que pueden estar obstaculizando una correcta orientación de la enseñanza. Cabe suponer, en efecto, que, si asumimos, por ejemplo, una visión individualista de la actividad científica, resultará difícil que los profesores concedamos al trabajo colectivo el papel que merece en la construcción de conocimientos científicos (Solomon, 1987; Linn, 1987; Burbules y Linn, 1991; Gil et al., 1991; Robinson y Niaz, 1991; Wheatley, 1991). Se ve así la conveniencia de sacar a la luz las posibles deformaciones que la enseñanza de las ciencias podría estar transmitiendo por acción u omisión. De este modo se favorecería el cuestionamiento de concepciones y prácticas asumidas acríticamente -por impregnación ambiental- y aproximarse a concepciones epistemológicas más correctas, susceptibles de incidir positivamente en el aprendizaje de las ciencias.

La investigación de estas deformaciones y de su mayor o menor incidencia puede realizarse de muy distintas maneras, que van desde el uso de cuestionarios al análisis de textos escolares o a la observación de las clases (Fernández, 2000). En este trabajo nos centraremos en el estudio de las posibles referencias a tales deformaciones en la literatura. Hemos procedido para ello a analizar los artículos aparecidos estos últimos diez años (desde 1990) en revistas como Science Education, International Journal of Science Education, Journal of Research in Science Teaching, Studies in Science Education, Science \& Education, Enseñanza de las Ciencias, etc., así como trabajos recogidos en el International Handbook of Science Education, editado por Fraser y Tobin (1998) y en la recopilación de McComas (1998) a la que 
hemos hecho referencia en el primer apartado. Así mismo hemos prestado atención a otros estudios anteriores a esta última década abundantemente citados que, por su importancia, nos ha parecido conveniente incluir.

En cada uno de los trabajos analizados, hemos retenido cualquier referencia a posibles reduccionismos y distorsiones de la actividad científica. Cabe señalar que, a menudo, estas referencias son breves e incidentales (es decir, no se trata de trabajos destinados expresamente a estudiar las concepciones epistemológicas de los profesores de ciencias ni las visiones deformadas de la ciencia que la enseñanza transmite), pero contribuyen, de todos modos, a evidenciar la extensión de la preocupación por su existencia.

Antes de pasar a detallar cuáles son las visiones deformadas mencionadas en la literatura, queremos llamar la atención sobre el hecho de que dichas deformaciones no pueden contemplarse como concepciones desligadas, autónomas; por el contrario, resulta lógico suponer que, si existe una serie de visiones deformadas acerca de la ciencia, dichas visiones no constituirán concepciones absolutamente autónomas, sino que, al igual que se ha mostrado en el caso de las preconcepciones de los estudiantes en un determinado dominio (Driver y Oldham, 1986), formarán un esquema conceptual relativamente integrado. Insistiremos en ello, utilizando ejemplos concretos, en la pequeña recapitulación con la que concluiremos este trabajo, pero hemos querido advertir aquí, de entrada, que la presentación correlativa que haremos de las distintas deformaciones mencionadas en la literatura no presupone una visión inconexa de las mismas.

Nos referiremos, a continuación, a las distintas deformaciones estudiadas, aportando en cada caso el correspondiente listado de referencias bibliográficas.

\section{Una concepción empiroinductivista y ateórica}

Como ya hemos señalado en el primer apartado, quizás la deformación que ha sido estudiada en primer lugar, y la más ampliamente señalada en la literatura, es la que podríamos denominar una concepción empiro-inductivista y ateórica de la ciencia. Una concepción que resalta el papel de la observación y de la experimentación «neutras» (no contaminadas por ideas apriorísticas), e incluso del puro azar, olvidando el papel esencial de las hipótesis como focalizadoras de la investigación y de los cuerpos coherentes de conocimientos (teorías) disponibles, que orientan todo el proceso.

Estas concepciones empiroinductivistas de la ciencia afectan a los científicos (Glasson y Bentley, 2000) pues, como explica Mosterín (1990), sería ingenuo pensar que «son siempre explícitamente conscientes de los métodos que usan en su investigación»-así como, lógicamente, a los estudiantes (Gaskell, 1992; Pomeroy, 1993; Roth y Roychondhury, 1994; Solomon, Duveen y Scott, 1994; Abrams y Wanderse, 1995; Traver, 1996; Roth y Lucas, 1997; Désautels y Larochelle, 1998b).
Cabe señalar que, aunque ésta es, sin duda, la deformación más estudiada y criticada en la literatura, tanto en la década de los años setenta como en el período 19842000 analizado (con más de sesenta trabajos que hacen referencia a la misma, que incluiremos más abajo) sigue siendo una concepción ampliamente extendida entre el profesorado de ciencias (Fernández, 2000). Es preciso tener en cuenta a este respecto que, pese a la importancia dada (verbalmente) a la observación y la experimentación, en general la enseñanza es puramente «libresca», sin apenas trabajo experimental. Ello favorece que la experimentación conserve para profesores y estudiantes el atractivo de una «revolución pendiente», como hemos podido percibir en entrevistas realizadas a profesores en activo (Fernández, 2000). De hecho, los intentos de renovación de la educación científica iniciados en los años sesenta incurrían, en sus mismas denominaciones - aprendizaje por descubrimiento...-en esta visión ateórica, centrada en el supuesto «método científico», con olvido de los contenidos (Gil, 1983; Phillps, 1985; Driver, 1983; Millar y Driver, 1987; Millar, 1988; Millar y Wynne, 1988; Gómez, Izquierdo y Sanmartí, 1990).

Cuadro I

Artículos que contemplan la concepción empiroinductivista y ateórica de la ciencia.

Nadeau y Dèsautels (1984); Hodson (1985); Otero (1985); Bronowski (1987); Giordan y De Vecchi (1987); Gould (1987); Selley (1989); Brickhouse (1989); Jacoby y Spargo (1989); Brickhouse (1990); Cleminson (1990); Koballa, Crawley y Shrigley (1990); Burbules y Linn (1991); Gallagher (1991); King (1991); Loving (1991); Matthews (1991); Solomon (1991); Gaskell (1992); Hodson (1992a); Lederman (1992); Linder (1992); Stinner (1992); Tobin, Tippins y Gallard, (1994); Carrascosa et al. (1993); Désautels et al. (1993); Gil (1993); Guilbert y Meloche (1993); Hodson (1993); Pomeroy (1993); Ruba y Harknerss (1993); Ruggieri, Tarsitani y Vicentini (1993); Acevedo (1994); Brickhouse (1994); Duschl (1994); Furió(1994); Gil(1994a); Lakin y Wellington (1994); Praia y Cachapuz, 1994a, 1994b; Roth y Roychoudhury (1994); Solomon, Duveen y Scott (1994); Tobin, Tippins y Hook, (1994); Cachapuz (1995b); Fernández y Orozco (1995); Hewson, Kerby y Cook (1995); Jiménez (1995); Koulaidis y Ogborn (1995); Orozco y Fernández (1995); Praia (1995); Gil (1996); Hashweeh (1996); Tomaz et al. (1996); Campos y Cachapuz (1997); Praia y Marques (1997); Boersema (1998); Cobern y Loving (1998); Dawkins y Glathorn (1998); Hammerich (1998); Lederman y Abd-El-Khalick (1998); Matson y Parsons (1998); McComas (1998a, 1998b); McComas y Olson (1998); McComas, Clough y Almazroa (1998); Meitchtry (1998); Nott y Wellington (1998); Paixão y Cachapuz (1998); Porlán y Rivero, (1998); Porlán et al. (1998); Praia y Cachapuz (1998); Spector, Strong y La Porta (1998); Sutton (1998); Yerrick, Pedersen y Arnason (1998); Lederman (1999); Lemberger, Hewson y Park (1999); Meitchtry (1999); Paixão y Cachapuz (1999); Praia y Coelho (1999); Sanmartí y Tarin (1999); Sperandeo-Mineo (1999); Cachapuz et al. (2000); Cachapuz, Praia y Jorge (2000a, 2000b); Cobern (2000); Glasson y Bentley (2000); Irwin (2000); Medeiros y Filho (2000); Paixão y Cachapuz (2000 a, 2000b); Campario, Moya y Otero, (2001), Paixão y Cachapuz (2001). 
Como prueba de la atención prestada por los investigadores a esta deformación, podemos dar la lista de trabajos del cuadro I (presentados por orden cronológico).

\section{Una concepción rígida de la actividad científica}

Una segunda deformación ampliamente recogida en la literatura (cerca de cuarenta artículos en el período analizado cuyas referencias se incluyen más abajo) es la que transmite una visión rígida (algorítmica, exacta, infalible...) de la actividad científica. Se presenta el «método científico» como un conjunto de etapas a seguir mecánicamente. Se resalta, por otra parte, lo que supone tratamiento cuantitativo, control riguroso, etc., olvidando -o, incluso, rechazando- todo lo que significa invención, creatividad, duda... Ello se pone particularmente en evidencia en lo que respecta a la evaluación: como afirma Hodson (1992b), la preocupación obsesiva por evitar la ambigüedad y asegurar la fiabilidad de las evaluaciones distorsiona la naturaleza misma del trabajo científico, esencialmente difuso, incierto, intuitivo... La evaluación debería tener en cuenta dicha ambigüedad, no intentar eliminarla.

Se trata de una concepción ampliamente difundida entre el profesorado de ciencias, como hemos podido constatar utilizando diversos diseños experimentales (Fernández, 2000). Así, en las entrevistas que hemos mantenido con profesores, una mayoría se ha referido al método científico como una secuencia de etapas definidas, destacando el rigor del mismo y el carácter exacto de los resultados obtenidos. Resultados semejantes han sido obtenidos por otros investigadores, pero cabe señalar que ésta es una deformación criticada por un cierto sector de los docentes, hasta el punto de que algunos, al rechazar esta visión rígida y dogmática de la ciencia, hacen suyo un relativismo extremo, tanto metodológico («todo vale», no hay estrategias específicas en el trabajo científico) como conceptual (no hay una realidad objetiva que permita contrastar la validez de las construcciones científicas: la única base en la que se apoya el conocimiento es el consenso de la comunidad de investigadores en ese campo); un relativismo del que encontramos apoyos en la literatura (Feyerabend, 1989) y, por supuesto, críticas (Izquierdo, Sanmartí y Espinet, 1999; Cobern, 2000).

Entre los trabajos que estudian o al menos abordan esta deformación podemos citar los que se incluyen el cuadro II.

\section{Una concepción aproblemática y ahistórica de la ciencia}

Muy ligada a esa visión rígida a la que acabamos de referirnos, podemos mencionar la visión aproblemática y ahistórica (ergo dogmática y cerrada): se transmiten conocimientos ya elaborados, sin mostrar cuáles fueron los problemas que generaron su construcción, cuál ha sido su evolución, las dificultades, etc., ni mucho menos aún, las limitaciones del conocimiento científico actual
Cuadro II

Artículos que contemplan la concepción rígida, algorítmica de la ciencia.

Aikenhead (1984); Hodson (1985); Porlan (1989); Gallagher (1991); Gaskell (1992); Hodson (1992a, 1992b); Linder (1992); Tobin, Tippins y Gallard, (1994); Carrascosa et al. (1993); Gil (1993); Guilbert y Meloche (1993); Hodson (1993); Acevedo (1994); Brickhouse (1994); Furió (1994); Gil (1994a); Lakin y Wellington (1994); Pedrinaci (1994); Praia y Cachapuz, 1994a, 1994b; Solomon, Duveen y Scott (1994); Tobin, Tippins y Hook (1994); Fernández y Orozco (1995); Orozco y Fernández (1995); Praia (1995); Gil (1996); Hashweeh (1996); Tomaz et al. (1996); Praia y Marques (1997); Boersema (1998); Cobern y Loving (1998); Dawkins y Glathorn (1998); Hammerich (1998); Lederman y Abd-El-Khalick (1998); Matson y Parsons (1998); McComas (1998a, 1998b); McComas y Olson (1998); Meitchtry (1998); Nott y Wellington (1998); Paixão y Cachapuz (1998); Porlan y Rivero (1998); Porlan et al. (1998); Praia y Cachapuz (1998); Spector, Strong y La Porta (1998); Sutton (1998); Yerrick, Pedersen y Arnason (1998); Lederman (1999); Meitchtry (1999); Paixão y Cachapuz (1999); Sanmartí y Tarin (1999); Cachapuz et al. (2000); Cachapuz, Praia y Jorge (2000a, 2000b); Cobern (2000); Glasson y Benteley (2000); Irwin (2000); Paixão y Cachapuz (2000b); Campario, Moya y Otero (2001); Paixão y Cachapuz (2001).

o las perspectivas abiertas. Se pierde así de vista que, como afirma Bachelard (1938), «todo conocimiento es la respuesta a una cuestión», a un problema, lo que dificulta captar la racionalidad del proceso científico.

Se trata de una concepción que la enseñanza de la ciencia refuerza por omisión. En efecto, los profesores de ciencias, tanto al ser entrevistados como cuando resuelven distintos tipos de cuestiones relativas a la forma de introducir los conocimientos científicos, no hacen referencia a los problemas que están en el origen de la construcción de dichos conocimientos. Es decir, la visión que transmiten, en general, incurre por omisión en una visión aproblemática; y lo mismo se aprecia en los libros de texto (Fernández, 2000). Más de treinta y cinco artículos se refieren a esta concepción (cuadro III).

\section{Una concepción exclusivamente analítica}

Una deformación que apenas es mencionada por los equipos docentes y que ha sido escasamente tratada por la investigación (menos de una docena de artículos hacen alguna referencia a la misma) es la consistente en una visión exclusivamente analítica, que resalta la necesaria parcelación inicial de los estudios, su carácter acotado, simplificatorio, pero que olvida los esfuerzos posteriores de unificación y de construcción de cuerpos coherentes de conocimientos cada vez más amplios o el tratamiento de problemas «puente» entre distintos campos de conocimiento que pueden llegar a unirse, como ha ocurrido tantas veces. 
Cuadro III

Artículos que contemplan la visión aproblemática y ahistórica de la ciencia

Otero (1985); Gagliardi y Giordan (1986); Giordan y De Vecchi (1987); Porlan (1989); Cleminson (1990); Koballa, Crawley y Shrigley (1990); García Cruz (1991); Linder (1992); Stinner (1992); Tobin, Tippins y Gallard (1994); Carrascosa et al. (1993); Gil (1993); Guilbert y Meloche (1993); Acevedo (1994); Brickhouse (1994); Furió (1994); Gil (1994a); Lakin, y Wellington (1994); Pedrinaci (1994); Praia y Cachapuz (1994a, 1994b); Roth y Roychoudhury (1994); Solomon, Duveen y Scott (1994); Tobin, Tippins y Hook (1994); Abrams y Wandersee (1995); Cachapuz (1995b); Fernández y Orozco (1995); Orozco y Fernández (1995); Praia (1995); Gil (1996); Hashweeh (1996); Tomaz et al. (1996); Campos y Cachapuz (1997); Boersema (1998); Dawkins y Glathorn (1998); Matson y Parsons (1998); McComas (1998a, 1998b); McComas y Olson (1998); Meitchtry (1998); Paixão y Cachapuz (1998); Porlán y Rivero (1998); Porlan et al. (1998); Sutton (1998); Yerrick, Pedersen y Arnason (1998); Lederman (1999); Meitchtry (1999); Praia y Coelho (1999); Sanmartí y Tarin (1999); Sperandeo-Mineo (1999); Cachapuz et al. (2000); Cachapuz, Praia y Jorge (2000a, 2000b); Cobern (2000); Glasson y Bentley (2000); Irwin (2000); Paixão y Cachapuz (2000a, 2000b); Teodoro y Nardi (2000); Campario, Moya y Otero (2001); Paixão y Cachapuz (2001).

Quizás esta escasa atención a una deformación que nos parece particularmente grave pueda ser debida a que las propuestas de tratamiento interdisciplinar e incluso de enseñanza integrada de las ciencias han sido ampliamente difundidas y parecen gozar de una buena aceptación (al menos verbal) entre los profesores. A nuestro entender, sin embargo, dichas propuestas incurren a menudo en un error de signo contrario al de la visión analítica, pero no menos grave, consistente en tomar la unidad de la materia como punto de partida, olvidándose que el

Cuadro IV

Artículos que contemplan la visión exclusivamente analítica de la ciencia.

Matthews (1991); Hodson (1992a); Carrascosa et al. (1993); Gil (1993); Furió (1994); Gil (1994a); Fernández y Orozco (1995); Orozco y Fernández (1995); Praia (1995); Gil (1996); McComas y Olson (1998); Spector, Strong y La Porta (1998); Lemberger, Hewson y Park (1999); Meitchtry (1999); Paixão y Cachapuz (1999); Paixão y Cachapuz (2000b).

establecimiento de dicha unidad constituye una conquista reciente y nada fácil de la ciencia (Gil et al., 1991; Gil, 1994b). Recordemos, por ejemplo, la fuerte oposición a las concepciones unitarias en astronomía (heliocentrismo), biología (evolucionismo) o en química orgánica (síntesis orgánica).

En cualquier caso, el olvido de los procesos de unificación como característica fundamental de la evolución de los conocimientos científicos constituye un auténtico obstáculo en la educación científica habitual. En efecto, hemos podido constatar (Fernández, 2000) que más de un $80 \%$ de los profesores y de los libros de texto incurre, por omisión, en esta deformación, olvidando destacar, por ejemplo, la unificación que supuso la síntesis newtoniana de las mecánicas celeste y terrestre, rechazada durante más de un siglo con condenas a la obra de Copérnico o Galileo.

Entre los escasos trabajos en los que esta deformación es mencionada podemos citar los que recoge el cuadro IV.

\section{Una concepción meramente acumulativa del desarrollo científico}

Una deformación a la que tampoco hacen referencia, a menudo, los equipos docentes -y que es la segunda menos mencionada en la literatura tras la visión exclusi-

Cuadro V

Artículos que contemplan la visión meramente acumulativa de la ciencia.

Porlan (1989); Cleminson (1990); Fillon (1991); Carrascosa et al. (1993); Gil (1993); Guilbert y Meloche (1993); Ruggieri, Tarsitani y Vicentini (1993); Brickhouse (1994); Furió (1994); Gil (1994a); Pedrinaci (1994); Cachapuz (1995b); Fernández y Orozco (1995); Orozco y Fernández (1995); Praia (1995); Gil (1996); Hashweeh (1996); Tomaz et al. (1996); Boersema (1998); Matson y Parsons (1998); McComas (1998a, 1998b); McComas y Olson (1998); McComas, Clough y Almazroa (1998); Meitchtry (1998); Nott y Wellington (1998); Porlán y Rivero, (1998); Spector, Strong y La Porta (1998); Izquierdo, Paixão y Cachapuz (1999); Praia y Coelho (1999); Sanmartí y Espinet (1999); Meitchtry (1999); Cachapuz et al. (2000); Cachapuz, Praia y Jorge (2000a, 2000b); Irwin (2000); Paixão y Cachapuz (2000b); Campario, Moya y Otero (2001).

vamente analítica-es la que transmite una visión acumulativa, de crecimiento lineal de los conocimientos científicos: el desarrollo científico aparece como fruto de un crecimiento lineal, puramente acumulativo (Izquierdo, Sanmartí y Espinet, 1999), ignorando las crisis y las remodelaciones profundas, fruto de procesos complejos que no se dejan ahormar por ningún modelo definido de cambio científico (Giere, 1998; Estany, 1990).

Esta deformación es complementaria, en cierto modo, de lo que hemos denominado visión rígida, aunque deben ser diferenciadas: mientras la visión rígida o algorítmica se refiere a cómo se concibe la realización de una investigación dada, la visión acumulativa es una inter- 
pretación simplista de la evolución de los conocimientos científicos a la que la enseñanza suele contribuir al presentar los conocimientos hoy aceptados sin mostrar cómo dichos conocimientos han sido alcanzados, ni referirse a las frecuentes confrontaciones entre teorías rivales, ni a los complejos procesos de cambio. Hemos encontrado referencias a esta deformación en los trabajos recogidos en el cuadro $\mathrm{V}$.

\section{Una concepción individualista y elitista de la ciencia}

Una de las deformaciones más tratadas en la literatura es la que transmite una visión individualista y elitista de la ciencia. Los conocimientos científicos aparecen como obra de genios aislados, ignorándose el papel del trabajo colectivo, de los intercambios entre equipos... En particular se deja creer que los resultados obtenidos por un sólo científico o equipo pueden bastar para verificar o falsar una hipótesis o, incluso, toda una teoría.

A menudo se insiste explícitamente en que el trabajo científico es un dominio reservado a minorías especialmente dotadas, transmitiendo expectativas negativas hacia la mayoría de los alumnos, con claras discriminaciones de naturaleza social y de género (la ciencia es presentada como una actividad eminentemente «masculina»).

Se contribuye, además, a este elitismo escondiendo la significación de los conocimientos tras presentaciones exclusivamente operativistas. No se realiza un esfuerzo por hacer la ciencia accesible (comenzando con tratamientos cualitativos, significativos), ni por mostrar su carácter de construcción humana, en la que no faltan confusiones ni errores, como los de los propios alumnos.

Cuadro VI

Artículos que contemplan la visión individualista y elitista de la ciencia

Aikenhead (1984); Gagliardi y Giordan (1986); Penick y Yager (1986); Cleminson (1990); Hodson (1992a, 1992b); Newton y Newton (1992); Stinner (1992); Carrascosa et al. (1993); Gil (1993); Guilbert y Meloche (1993); Ruggieri, Tarsitani y Vicentini (1993); Brickhouse (1994); Furió (1994); Gil (1994a); Lakin y Wellington (1994); Tobin, Tippins y Hook (1994); Abrams y Wandersee (1995); Fernández y Orozco (1995); Hewson, Kerby y Cook (1995); Orozco y Fernández (1995); Praia (1995); Gil (1996); Tomaz et al. (1996); Matthews (1997); Roth y Lucas (1997); Boersema (1998); Cobern y Loving (1998); Dawkins y Glathorn (1998); Hammerich (1998); Lederman y AbdEl-Khalick (1998); Matson y Parsons (1998); McComas (1998a, 1998b); McComas y Olson (1998); Meitchtry (1998); Nott y Wellington (1998); Porlán y Rivero (1998); Spector, Strong y La Porta (1998); Sutton (1998); Yerrick, Pedersen y Arnason (1998); Meitchtry (1999); Paixão y Cachapuz (1999); Praia y Coelho (1999); Sanmartí y Tarin (1999); Paixão y Cachapuz (2000b); Campario, Moya y Otero (2001).
En algunas ocasiones nos encontramos con una deformación de signo opuesto que contempla la actividad científica como algo sencillo, próximo al sentido común, olvidando que la construcción científica parte, precisamente, del cuestionamiento sistemático de lo obvio (Bachelard, 1938).

Referencias a esta extendida concepción las hemos encontrado en los trabajos incluidos en el cuadro VI.

\section{Una visión descontextualizada, socialmente neutra de la actividad científica}

Por último nos referiremos a la deformación que transmite una visión descontextualizada, socialmente neutra de la ciencia que ignora, o trata muy superficialmente, las complejas relaciones CTS, ciencia-tecnologíasociedad (o, mejor, CTSA, agregando la A de ambiente para llamar la atención sobre los graves problemas de degradación del medio que afectan a la totalidad del planeta). De hecho, las referencias más frecuentes a las relaciones CTSA que incluyen la mayoría de los textos escolares de ciencias se reducen a la enumeración de algunas aplicaciones de los conocimientos científicos (Solbes y Vilches, 1997), cayendo así en una exaltación simplista de la ciencia como factor absoluto de progreso.

Frente a esta ingenua visión de raíz positivista, comienza a extenderse una tendencia a descargar sobre la ciencia y la tecnología la responsabilidad de la situación actual de deterioro creciente del planeta, lo que no deja de ser una nueva simplificación maniquea en la que resulta fácil caer y que llega a afectar, incluso, a algunos libros de texto (Solbes y Vilches, 1998). No podemos ignorar, a este respecto, que son científicos quienes estudian los problemas a que se enfrenta hoy la humanidad, advierten de los riesgos y ponen a punto soluciones (Sánchez Ron, 1994). Por supuesto, no sólo los científicos ni todos los científicos. Es cierto que son también científicos y tecnólogos quienes han producido, por ejemplo, los compuestos que están destruyendo la capa de ozono, pero junto a economistas, políticos, empresarios y trabajadores. Las críticas y las llamadas a la responsabilidad han de extenderse a todos, incluidos los «simples» consumidores de los productos nocivos.

Cabe señalar, sin embargo, que estas actitudes simplistas de exaltación beata o de rechazo absoluto de la ciencia son minoritarias: lo más frecuente es que se incurra en visiones puramente operativistas que ignoran completamente la contextualización de la actividad científica, como si la ciencia fuera un producto elaborado en torres de marfil, al margen de las contingencias de la vida ordinaria y proporciona una imagen de los científicos como seres «por encima del bien y del mal», encerrados en torres de marfil y ajenos a las necesarias tomas de decisión.

Aunque en los últimos años los medios de comunicación se hacen frecuentemente eco de noticias acerca de, por ejemplo, los problemas del medio ambiente provocados 
Cuadro VII

Artículos que contemplan la visión descontextualizada de la ciencia.

Aikenhead (1984); Gagliardi y Giordan (1986); Brush (1989); Cleminson (1990); García Cruz (1991); Gaskell (1992); Hodson (1992a, 1992b); Linder (1992); Carrascosa et al. (1993); Gil (1993); Guilbert y Meloche (1993); Ruba y Harknerss (1993); Ruggieri, Tarsitani y Vicentini (1993); Acevedo (1994); Brickhouse (1994); Furió (1994); Gil (1994a); Abrams y Wandersee (1995); Fernández y Orozco (1995); Orozco y Fernández (1995); Gil (1996); Tomaz et al. (1996); Campos y Cachapuz (1997); Matthews (1997); Boersema (1998); Cobern y Loving (1998); Dawkins y Glatthorn (1998); Lederman y Abd-El-Khalick (1998); Matson y Parsons (1998); McComas (1998a, 1998b); McComas y Olson (1998); McComas, Clough y Almazroa (1998); Meitchtry (1998); Nott y Wellington (1998); Paixão y Cachapuz (1998); Porlán y Rivero, (1998); Spector, Strong y La Porta (1998); Sutton (1998); Yerrick, Pedersen y Arnason (1998); Lederman (1999); Meitchtry (1999); Praia y Coelho (1999); Sanmartí y Tarin (1999); Cachapuz et al. (2000); Cachapuz, Praia y Jorge (2000a, 2000b); Glasson y Bentley (2000); Paixão y Cachapuz (2000b); Paixão y Cachapuz (2001).

por determinados desarrollos científicos no sometidos al «principio de prudencia», hemos podido constatar que un elevado porcentaje de profesores no toma en consideración esta dimensión de la actividad científica. Ello es particularmente notorio en lo que se refiere a las actividades utilizadas para evaluar el aprendizaje, lo que en definitiva muestra la escasa importancia que se le concede (Alonso, Gil y Martínez Torregrosa, 1992).

Entre los trabajos que se refieren a este peligro de descontextualización de la actividad científica, cabe mencionar los incluidos en el cuadro VII.

\section{CONCLUSIÓN}

Éstas son, en síntesis las siete grandes deformaciones que hemos visto tratadas en la literatura, aunque la frecuencia con la que los investigadores prestan atención a cada una de ellas es desigual, como puede verse en el cuadro VIII. Son también las deformaciones que hemos visto reflejadas en la docencia habitual en un estudio detenido que ha utilizado cerca de veinte diseños experimentales (Fernández, 2000).

Como ya hemos advertido, estas deformaciones no constituyen una especie de «siete pecados capitales» distintos y autónomos, sino que se apoyan mutuamente y forman un esquema conceptual relativamente integrado. En efecto, es lógico que una visión individualista y elitista de la ciencia apoye implícitamente la idea empirista de descubrimiento y contribuya, además, a una lectura descontextualizada, socialmente neutra, de la actividad científica (realizada por «genios» solitarios). Del mismo modo, por citar otro ejemplo, una visión rígida, algorítmica, exacta de la ciencia puede reforzar una interpretación acumulativa, lineal, del desarrollo científico, ignorando las crisis y las revoluciones científicas.

Así pues, estas concepciones aparecen asociadas entre sí, como expresión de una imagen ingenua de la ciencia que se ha ido decantando, pasando a ser socialmente aceptada. De hecho esa imagen tópica de la ciencia parece haber sido asumida incluso por numerosos autores del campo de la educación, que critican como características de la ciencia lo que no son sino visiones deformadas de la misma. Así, por ejemplo, Kemmis y McTaggert (1982) (citado por Hodson, 1992a) critican a la «investigación convencional» (o «académica») su carácter «neutral», su preocupación exclusiva por «acumular conocimientos» (sin atención a «la mejora de la práctica»), su limitación a «un mero procedimiento de resolución de problemas» (olvidando el planteamiento de los mismos), etc. Kemmis y McTaggert insisten reiteradamente en su crítica a la investigación académi$\mathrm{ca}$, atribuyéndole deformaciones y reduccionismos que los autores dan por sentado que corresponden al «método científico» utilizado por «las ciencias naturales».

Incluso entre algunos investigadores en didáctica de la ciencia parece aceptarse que la ciencia clásica sería puramente analítica, «neutra», etc. Ya no se trata de que la enseñanza haya transmitido esas concepciones reduccionistas, empobrecedoras, sino que toda la ciencia clásica tendría esos defectos (García, 1995).

Pero, ¿cómo se puede afirmar que la ciencia clásica es -como suele afirmarse- puramente analítica, si su primer edificio teórico significó la integración de dos universos considerados esencialmente distintos, derribando la supuesta barrera entre el mundo celeste y el sublunar? Una integración, además, que implicaba desafiar dogmas, tomar partido por la libertad de pensamiento, correr riesgos de condenas, de inclusión en el Index Librorum

\section{Cuadro VIII}

Frecuencia con la que se menciona cada visión simplista en la literatura consultada.

\begin{tabular}{|l|l|l|l|l|l|l|l|}
\hline Visiones simplistas & a & b & c & d & e & f & g \\
\hline Frecuencia & 92 & 61 & 61 & 16 & 38 & 47 & 51 \\
\hline
\end{tabular}

a) Visión empiroinductivista, ateórica

b) Visión rígida (algorítmica, exacta, infalible...)

c) Visión aproblemática y ahistórica (ergo dogmática y cerrada)

d) Visión exclusivamente analítica

e) Visión acumulativa, de crecimiento lineal

f) Visión individualista y elitista

g) Visión socialmente descontextualizada 
Prohibitorum... y otros más graves. Y no es sólo la mecánica: toda la ciencia clásica puede interpretarse como la superación de supuestas barreras, la integración de dominios separados (por el sentido común y por los dogmas). Pensemos en la teoría de la evolución de las especies; en la síntesis orgánica (¡en el siglo xix todavía se sostenía la existencia de un elan vital y se negaba la posibilidad de sintetizar compuestos orgánicos!); en el electromagnetismo que mostró los vínculos entre electricidad, magnetismo y óptica; en los principios de conservación y transformación de la masa y de la energía, aplicables a cualquier proceso (Gil et al., 1991). ¿Dónde está el carácter puramente analítico? ¿Dónde está el carácter neutro, aséptico, de esa ciencia? Hay que reconocer que, al menos, no toda la ciencia clásica ha sido así. Parece más apropiado, pues, hablar de visiones (o, en todo caso, tendencias) deformadas de la ciencia, que atribuir esas características a toda la ciencia clásica.

Las concepciones docentes sobre la ciencia serían, pues, expresión de esa visión común, que los profesores de

\section{REFERENCIAS BIBLIOGRÁFICAS}

ABRAMS, E. y WANDERSEE, J.H. (1995). How to infuse actual scientific research practices into science classroom instruction. International Journal of Science Education, 17(6), pp. 683-694.

ACEVEDO, J.A. (1994). Los futuros profesores de enseñanza secundaria ante la sociología y la epistemología de las ciencias: Un enfoque CTS. Revista Interuniversitaria de Formación del Profesorado, 19, pp. 111-125.

AIKENHEAD, G.S. (1984). Teacher decision making: The case of Prairie High. Journal of Research in Science Education, 21, pp. 167-186.

ALONSO, S.M., GIL, D. y MARTÍNEZ TORREGROSA, J. (1992). Concepciones espontáneas de los profesores de Ciencias sobre la evaluación. Revista de Enseñanza de la Física, 5(2), pp. 18-38.

BACHELARD, G. (1938). La Formation de l'esprit scientifique. París: Vrin.

BARROW,D.A. (1991). Critical reflection: A Source of Wonderful Ideas for Changing Classroom Practices. Journal of Elementary Science Education, 3(2), pp. 26-39.

BELL, B. F. y PEARSON, J. (1992). Better Learning. International Journal of Science Education, 14(3), pp. 349-361. ciencias aceptaríamos implícitamente debido a la falta de reflexión crítica y a una educación científica que se limita, a menudo, a una simple transmisión de conocimientos ya elaborados. Ello no sólo deja en la sombra las características esenciales de la actividad científica, sino que contribuye a reforzar algunas deformaciones, como el supuesto carácter «exacto» (ergo dogmático) de la ciencia o la visión aproblemática, etc. De este modo, la imagen de la ciencia que poseen los docentes se diferenciaría poco de la que puede expresar cualquier ciudadano y resulta muy alejada de las concepciones actuales acerca de la naturaleza de la ciencia. De ahí la importancia de un trabajo de clarificación que permita a los docentes distanciarse críticamente de estas visiones deformadas. Como señalan Guilbert y Meloche (1993), «Una mejor comprensión por los docentes de los modos de construcción del conocimiento científico [...] no es únicamente un debate teórico, sino eminentemente práctico». La presente revisión bibliográfica pretende contribuir a mostrar la importancia de esta tarea y proporcionar documentación útil para los necesarios esfuerzos de formación docente a este respecto.
BOERSEMA, D. (1998). The use of real and imaginary cases in communicating the nature of science course outline, en McComas, W.F. (ed.). The nature of science in science education. Rationales and strategies, pp. 255-266. Netherlands: Kluwer Academic Publishers.

BRICKHOUSE, N.W. (1989). The teaching of the philosophy of science in secondary classrooms: Case studies of teachers' personal theories. International Journal of Science Education, 11, pp. 437-449.

BRICKHOUSE, N.W. (1990). Teachers' beliefs about the nature of science and their relationship to classroom practice. Journal of Teacher Education, 41(3), pp. 53-62.

BRICKHOUSE, N.W. (1994). Children's observations, ideas and the development of classroom. Theories about light. Journal of Research in Science Teaching, 31(6), pp. 639656.

BRONOWSKI, J. (1987). The creative process, en Scientific Genius and Creativity: Readings from Scientific American. pp. 1-8. Nueva York: Freeman.

BRUSH, S.G. (1989). History of science and science education. Interchange, 20, pp. 60-71.

BURBULES, N. y LINN, M. (1991). Science education and philosophy of science: congruence or contradiction? International Journal of Science Education, 13(3), pp. 227-241. 
CACHAPUZ, A.F. (1995a). Da investigação sobre e para professores à investigação com e pelos professores de ciências, en La formación del profesorado de ciencias y matemáticas en España y Portugal, pp. 243-254. Departamento de Didáctica de las Ciencias Experimentales y de las Matemáticas. Facultad de Educación. Universidad de Extremadura. Badajoz.

CACHAPUZ, A. (1995b). O Ensino das Ciências para a Excelência das Aprendizagens, en De Carvalho, A.D. (org.). Novas Metodologias em Educação, pp. 349-385. Porto: Porto Editora.

CACHAPUZ, A., PRAIA, J., PAIXÃO, F. y MARTINS, I. (2000). Uma visão sobre o ensino das ciências no pósmudança conceptual: contributos para a formação de professores. Inovação, 2-3, pp. 117-137.

CACHAPUZ, A., PRAIA, J. y JORGE, M. (2000a). Perspectivas de Ensino. Textos de Apoio ${ }^{\circ} 1$, en Formação de Professores /Ciências, A. Cachapuz (org.). Porto: Centro de Estudos em Educação em Ciência.

CACHAPUZ, A., PRAIA, J. y JORGE, M. (2000b). Reflexão em torno de perspectivas do ensino das ciências: contributos para uma nova orientação curricular-ensino por pesquisa. Revista de Educação, IX(1), pp. 69-79.

CAMPARIO, J.M., MOYA, A. y OTERO, J.C. (2001). Invocaciones y usos inadecuados de la ciencia en la publicidad. Enseñanza de las Ciencias, 19(1), pp. 45-56.

CAMPOS, C. y CACHAPUZ, A. (1997). Imagens de Ciência em manuais de química portugueses, Química Nova, 6, pp. 23-29.

CARRASCOSA, J. et al. (1993). Los programas de formación permanente del profesorado de física y química en la Comunidad Valenciana: Un intento constructivista de formación didáctica. Enseñanza de las Ciencias, núm. extra, pp. 47-48.

CLEMINSON, A. (1990). Establishing an epistemological base for science teaching in the light of contemporary notions of the nature of science and of how children learn science. Journal of Research in Science Teaching, 27(5), pp. 429- 445.

COBERN, W.W. (2000). The nature of science and the role of knowledge and belief. Science \& Education, 9(3), pp. 219-246.

COBERN, W.W. y LOVING, C. (1998). The card exchange introducing the philosophy of science, en McComas, W.F. (ed.). The nature of science in science education. Rationales and strategies, pp. 73-82. Netherlands: Kluwer Academic Publishers.

CRONIN-JONES, L. (1991). Science teaching beliefs and their influence on curriculum implementations: Two case studies. Journal of Research in Science Teaching, 38(3), pp. 235-250.

DAWKINGS, K.R. y GLATTHORN, A.A. (1998). Using historical case studies in biology to explore the nature of science: A professional development program for high school teachers, en McComas, W.F. (ed.). The nature of science in science education. Rationales and strategies, pp. 163-176. Netherlands: Kluwer Academic Publishers.

DÉSAUTELS, J., LAROCHELLE, M., GAGNÉ, B y RUEL, F. (1993). La formation a l'enseignement des sciences: le virage épistémologique. Didaskalia, 1, pp. 49-67.

DÉSAUTELS, J. y LAROCHELLE, M. (1998a). About the epistemological posture of science teachers, en Tiberghien A., Jossem, L. y Barojas, J. (eds.). Connecting Research in Physics Education with Teacher Education (ICPE Books).
DÉSAUTELS, J. y LAROCHELLE, M. (1998b). The epistemology of students: The «thingified» nature of scientific knowledge. International Handbook of Science Education, pp. $115-126$.

DRIVER, R. (1983). The pupil as scientist? Milton Keynes, Open University.

DRIVER, R. y OLDHAM, V. (1986). A constructivist approach to curriculum development in science. Studies in Science Education, 13, pp. 105-122.

DUSCHL, R.A. (1994). Research on the history and philosophy of science, en Dorothy, L.G. (ed.). Handbook of Research on Science Teaching and Learning, pp. 445-455. Nueva York: McMillan.

ESTANY A. (1990). Modelos de cambio científico. Barcelona: Crítica.

FERNÁNDEZ, I. (2000). «Análisis de las concepciones docentes sobre la actividad científica: una propuesta de transformación». Tesis doctoral. Departamento de Didáctica de las Ciencias Experimentales y Sociales. Universidad de Valencia.

FERNÁNDEZ, I. y OROZCO, A. (1995). La transformación de las concepciones espontáneas sobre la ciencia. Tesis de Tercer Ciclo. Universidad de Valencia.

FEYERABEND, P. (1989). Contra el método. Barcelona: Ariel.

FILLON, P. (1991). Histoire des sciences et réflexion épistémologiques des élèves. Aster, 12, pp. 91-120.

FURIÓ, C. (1994). Tendencias actuales en la formación del profesorado de ciencias. Enseñanza de las Ciencias, 12(2), pp. 188-199.

GAGLIARDI, R. y GIORDAN, A. (1986). La historia de las ciencias: Una herramienta para la enseñanza. Enseñanza de las ciencias, 4(3), pp. 253-258.

GALLAGHER, J.J. (1991). Perspective and Practising Secondary School Science Teachers Knowledge and Beliefs about the Philosophy of Science. Science Education, 75(1), pp. 121-133.

GARCÍA CRUZ, C.M. (1991). La historia de la ciencia en la futura enseñanza secundaria: Reflexiones en torno al diseño curricular base. Enseñanza de las Ciencias, 10(1), pp. 115-117.

GARCÍA DÍAZ, J.E. (1995). «Epistemología de la complejidad y enseñanza de la ecología. El concepto de ecosistema en la enseñanza secundaria». Tesis doctoral. Universidad de Sevilla, 1995.

GASKELL, P.J. (1992). Authentic science and school science. International Journal of Science Education, 14(3), pp. 265272.

GENÉ, A. y GIL, D. (1987). Tres principios básicos en el diseño de la formación del profesorado. Andecha Pedagógica, 18, pp. 28-30.

GIERE, R.N. (1988). Explaining Science. A cognitive approach. Chicago: The University of Chicago Press.

GIL, D. (1983). Tres paradigmas básicos en la enseñanza de la ciencia. Enseñanza de las Ciencias, 1(1), pp. 26-33.

GIL, D. (1993). Contribución de la historia y la filosofía de las ciencias al desarrollo de un modelo de enseñanza-aprendizaje como investigación. Enseñanza de las Ciencias, 11(2), pp. 197-212. 
GIL, D. (1994a). Diez años de investigación en didáctica de las ciencias: realizaciones y perspectivas. Enseñanza de las Ciencias, 12(2), pp. 154-164.

GIL,D. (1994b). El currículo de ciencias en la educación secundaria: ¿Área o disciplinas? ¡Ni lo uno ni lo otro, sino todo lo contrario! Infancia y Aprendizaje, 65, pp. 19-30.

GIL, D. (1996). New trends in science education. International Journal in Science Education, 18(8), pp. 889-901.

GIL, D., CARRASCOSA, J. FURIÓ, C., MARTÍNEZTORREGROSA, J. (1991). La enseñanza de las ciencias en la educación secundaria. ICEUniversidad de Barcelona. Barcelona: Horsori.

GIORDANA.(1978). Observation-Experimentation: mais comment les élèves apprennent-ils? Revue Francaise de Pedagogie, 44, pp. 66-73. Trad. española en Infancia y Aprendizaje, 1978, núm. 13.

GIORDAN, A. y DE VECCHI, G. (1987). Les origines du savoir. Des conceptions des apprenants aux concepts scientifiques. Neuchatel: Delachauz \& Niestlé. Trad. cast. Los orígenes del saber. De las concepciones personales a los conceptos científicos. Sevilla: Díada, 1998.

GLASSON, G.E. y BENTELEY, M.L. (2000). Epistemological undercurrents in scientists' reporting of research to teachers. Science Education, 84(4), pp. 469-485.

GÓMEZ, I., IZQUIERDO, M. y SANMARTÍ, N. (1990). Los procedimientos. Cuadernos de Pedagogía, 180, pp. 28-31.

GOULD, S.J. (1987). Time's arrow time's cycle. Cambridge: Harvard University Press.

GUILBERT, L. y MELOCHE, D. (1993). L'idée de science chez des enseignants en formation: un lieu entre l'histoire des sciences et l'hétérogénéité des visions? Didaskalia, 2, pp. 730 .

HAMMERICH, P.L. (1998). Confronting students' conceptions of the nature of science with co-operative controversy, en McComas, W.F.(ed.). The nature of science in science education. Rationales and strategies, pp. 127-133. Netherlands: Kluwer Academic Publishers.

HASHWEEH, M.Z. (1996). Effects of science teachers' epistemological beliefs in teaching. Journal of Research in Science Teaching, 33 (1), pp. 47-63.

HEWSON, P.W. y HEWSON M.G. (1987). Science teacher's conceptions of teaching: Implications for teacher education. International Journal of Science Education, 9, pp. 425-440.

HEWSON,P.W., KERBY,H.W.yCOOK,P.A.(1995). Determining the conceptions of teaching science held by experienced high school science teachers. Journal of Research in Science Teaching, 32(5), pp. 503-520.

HODSON, D. (1985). Philosophy of science, science and science education. Studies in Science Education, 12, pp. 25-57.

HODSON, D. (1992a). In search of a meaningful relationship: an exploration of some issues relating to integration in science and science education. International Journal of Science Education, 14, pp. 541-562.

HODSON, D. (1992b). Assessment of practical work: some considerations in philosophy of science. Science Education, 1(2), pp. 115-144.

HODSON, D. (1993). Philosophy stance of secondary school science teachers, curriculum experiences and children's understanding of science: some preliminary findings. Interchange, 24(1-2), pp. 41-52.
IZQUIERDO, M., SANMARTÍ, N. y ESPINET, M. (1999). Fundamentación y diseño de las prácticas escolares de ciencias experimentales. Enseñanza de las Ciencias, 17(1), pp. 45-60.

IRWIN, A.R. (2000). Historical case studies: Teaching the nature of science in context. Science Education, 84(1), pp. $5-26$.

JACOBY, B.A. y SPARGO, P.E. (1989). Ptolemy revisited? The existence of a mild instrumentalism in some selected high school physical science textbooks. Interchange, 20, pp. 33-53.

JIMÉNEZ ALEIXANDRE, M.P. (1995). La formación del profesorado de ciencias y matemáticas en España y Portugal. Publicación del Departamento de Didáctica de las Ciencias. Universidad de Extremadura.

KEMMIS, S. y McTAGGERT, R. (1982). The Action Research Planner. Geelong: Deakin University Press.

KING, B.B. (1991). Beginning Teachers Knowledge of and Attitude Towards History and Philosophy of Science. Science Education, 75(1), pp. 135-141.

KOBALLA, T.R., CRAWLEY, F.E. y SHRIGLEY, R.L. (1990). A summary of research in Science Education (1988). Science Education, 74(3), pp. 253-407.

KOULAIDIS, V. y OGBORN, J. (1995). Science teacher philosophical assumptions: how well do we understand them? International Journal Science Education, 17(3), pp. 273-283.

LAKIN, S. y WELLINGTON, J. (1994). Who will teach the «nature of science»? Teachers view of science and their implications for science education. International Journal of Science Education, 16(2), pp. 175-190.

LEDERMAN, N.G. (1992). Students' and teachers' conceptions of the nature of science: A review of the research. Journal of Research in Science Teaching, 29, pp. 331-359.

LEDERMAN, N.G. (1999). Teachers' understanding of nature of science and classroom practice: factors that facilitate or impede the relationship. Journal of Research in Science Teaching, 36(8), pp. 916-929.

LEDERMAN, N.G y ABD-EL-KHALICK, F. (1998). Avoiding de-natured science: Activities that promote understanding of the nature of science, en W.F. McComas (ed.). The nature of science in science education. Rationales and strategies, pp. 41-52. Netherlands: Kluwer Academic Publishers.

LEMBERGER, J., HEWSON, P.W. y PARK, H. (1999). Relationship between prospective secondary teachers' classroom practice and their conceptions of biology and teaching science. Science Education, 83(3), pp. 347-372.

LINDER, C.J. (1992). Is teacher-reflected epistemology a source of conceptual difficulty in physics? International Journal of Science Education, 14(1), pp. 111-121.

LINN, M. (1987). Establishing a research base for science education: Challenges, trends and recommendations. Journal of Research in Science Teaching, 24(3), pp. 191-216.

LOVING, C.C. (1991). The Scientific Theory Profile: A Philosophy of Science Models for Science Teacher. Journal of Research in Science Teaching, 28(9), pp. 823-838.

MATSON, J.O. y PARSON, S. (1998). The nature of science: achieving scientific literacy by doing science, en McComas, W.F. (ed.). The nature of science in science education. 
Rationales and strategies, pp. 223-230. Netherlands: Kluwer Academic Publishers.

MATTHEWS, M.R. (1991). Un lugar para la historia y la filosofía en la enseñanza de las ciencias. Comunicación, Lenguaje y Educación, 11-12, pp. 141-155.

MATTHEWS, M.R. (1997). Editorial. Science and Education, 6, pp. 323-329.

McCOMAS, W.F. (1998a). The principal elements of the nature of science: Dispelling the myths, en McComas (ed.). The nature of science in science education. Rationales and strategies, pp. 53-70. Netherlands: Kluwer Academic Publishers.

McCOMAS, W.F. (1998b). A thematic introduction to the nature of science: The rationale and content of a course for science educators, en McComas, W.F. (ed.). The nature of science in science education. Rationales and strategies, $\mathrm{pp}$. 211-222. Netherlands: Kluwer Academic Publishers.

McCOMAS, W.F., CLOUGH, M.P. y ALMAZROA, H. (1998). The role and character of the nature of science in science education, en W.F. McComas (ed.). The nature of science in science education. Rationales and strategies, pp. 3-39. Netherlands: Kluwer Academic Publishers.

McCOMAS, W.F. y OLSON, J. (1998). The nature of science in international science education standards documents, en W.F. McComas (ed.). The nature of science in science education. Rationales and strategies, pp. 41-52. Netherlands: KluwerAcademic Publishers.

MEDEIROS, A. y FILHO, S.B. (2000). A Natureza da Ciência e a Instrumentação para o Ensino da Física. Ciência \& Educação, 6(2), pp. 107-117.

MEICHTRY, Y.(1998). Elementary science teaching methods: Developing and measuring student' views about the nature of science, en McComas, W.F. (ed.). The nature of science in science education. Rationales and strategies, pp. 231241. Netherlands: Kluwer Academic Publishers.

MEICHTRY, Y. (1999). The nature of science and scientific knowledge: Implications for a preserve elementary methods course. Science \& Education, 8(3), pp. 273-286.

MILLAR, R. (1988). What is scientific method, and can it be taught?, en Wellington, J. (ed.). Skills and Processes in Science Education. A critical analysis, pp. 47-61. Nueva York: Routledge.

MILLAR, R. y DRIVER, R. (1987). Beyond processes. Studies in Science Education, 14, pp. 33-62.

MILLAR, R. y WYNNE, B. (1988). Public understanding of Science: from contents to processes. International Journal of Science Education, 10(4), pp. 388-398.

MOSTERÍN J. (1990). Prólogo al libro de Estany A. Modelos de cambio científico. Barcelona: Crítica.

NADEAU, R. y DÉSAUTELS, J. (1984). Epistemology and the teaching of science. Ottawa: Science Council of Canada.

NEWTON, D.P. y NEWTON, L.D. (1992). Young children's perceptions of science and scientist. International Journal in Science Education, 148(3), pp. 331-348.

NOTT, M. y WELLINGTON, J. (1998). A programme for developing understanding of the nature of science in teacher education, en McComas, W.F. (ed.). The nature of science in science education. Rationales and strategies, pp. 293313. Netherlands: Kluwer Academic Publishers.
NUSSBAUM, J. (1989). Classroom conceptual change: philosophical perspectives. International Journal in Science Education, 11, special issue, pp. 530-540.

OROZCO, A. y FERNÁNDEZ, I. (1995). «El problema de las concepciones espontáneas sobre la ciencia». Tesis de tercer ciclo. Universidad de Valencia: Valencia.

OTERO, J. (1985). Assimilation problems in traditional representation of scientific knowledge. European Journal of Science Education, 7(4), pp. 361-369.

PAIXÃO, M. F. (1998). «Da Construção do Conhecimento Didáctico na Formação de Professores de Ciências. Conservação da Massa nas Reacções Químicas: estudo de índole epistemológica». Tesis doctoral. Aveiro: Universidade de Aveiro.

PAIXÃO, M.F. y CACHAPUZ, A. (1998). Dimensión epistemológica de los programas de física y química e implicaciones en las prácticas de enseñanza: ¿Qué lectura hacen los profesores?, en Banet, E. y De Pro, A. (eds.). Investigación e Innovación en la Enseñanza de las Ciencias, 1, pp. 284- 293.

PAIXÃO, M.F. y CACHAPUZ, A. (1999). La enseñanza de las ciencias y la formación de profesores de enseñanza primaria para la reforma curricular. De la teoría a la práctica. Enseñanza de las Ciencias, 17(1), pp. 69-77.

PAIXÃO, M.F. y CACHAPUZ, A. (2000a). Mass Conservation in Chemical Reactions: the development of innovative teaching strategy based on the History and Philosophy of Sience. Chemistry Education. Research and Practic in Europe, 1(2), pp. 201-215.

PAIXÃO, M.F. y CACHAPUZ, A. (2000b). Challenges on science teacher education for the new century: an approach based on the epistemology of curricular themes. Proceeding Book, en la $24^{\text {th }}$ ATEE Anual Conference. Leipzig. http:// www.atee.org/htm/conferences/leipzig/abstracts/rdc2paixao.html

PAIXÃO, M.F. y CACHAPUZ, A. (2001). Formación epistemológica y cambio de imágenes de ciencia impartidas en el aula. Revista de Educación en Ciencias - Journal of Science Education, 2(1), pp. 33-38.

PEDRINACI, E. (1994). Epistemología, historia de las ciencias y abejas. Investigación en la Escuela, 23, pp. 95-102.

PENICK, J.E. y YAGER, R.E. (1986). Trends in science education: Some observations of exemplary programmes in the USA. European Journal of Science Education, 8, pp. 1-8.

PHILLIPS, D.C. (1985). Can scientific method be taught? Journal of Curriculum Studies Teaching, pp. 95-101.

POMEROY, D. (1993). Implications of teachers' beliefs about the nature of science: Comparison of the beliefs of scientits, secondary science teachers and elementary teachers. Science Education, 77(3), pp. 261-278.

PORLÁN, R. (1989). «Teoría del conocimiento, teoría de la enseñanza y desarrollo profesional. Las concepciones epistemológicas de los profesores». Tesis doctoral. Universidad de Sevilla.

PORLÁN, R. y RIVERO, A. (1998). El conocimiento de los profesores. Sevilla: Díada Editora.

PORLÁN, R., GARCÍA, E., RIVERO, A. y MARTÍN DEL POZO, R. (1998). Les obstacles a la formation profesionelle des professeurs en rapport avec leurs idées sur la science, l'enseignement et l'apprentissage. Aster, 26, pp. 207-235. 
PRAIA, J. (1995). «Formação de Professores no Ensino da Geologia: contributos para uma Didáctica fundamentada na epistemologia das ciências. O caso da Deriva Continental». Tesis doctoral. Universidade de Aveiro. Aveiro.

PRAIA, J. y CACHAPUZ, F. (1994a). Para uma reflexão em torno as concepções epistemológicas dos professores de Ciências dos Ensinos Básico ( $3^{\circ}$ ciclo) e Secundário. Revista Portuguesa de Educação, 7(1/2), pp. 37-47. Braga: Universidade do Minho.

PRAIA, J. y CACHAPUZ, A. (1994b). Un análisis de las concepciones acerca de la naturaleza del conocimiento científico de los profesores portugueses de la enseñanza secundaria. Enseñanza de las Ciencias, 12(3), pp. 350-354.

PRAIA, J. y CACHAPUZ, A. (1998). Concepções epistemológicas dos professores portugueses sobre o trabalho experimental. Revista Portuguesa de Educação, 11 (1), pp. 71-85.

PRAIA, J. y COELHO, J. (1999). A Epistemologia, a História e a Sociologia da Ciência na construção de materiais didácticos «A Origem da Vida». Revista de Educação, VIII(2), pp. 203-220. Departamento de Educação da Faculdade de Ciências de Lisboa.

PRAIA, J. y MARQUÉS, L. (1997). El trabajo de laboratorio en la enseñanza de la geología: reflexión crítica y fundamentos epistemológico-didácticos. Enseñanza de las Ciencias de la Tierra, 5(29), pp. 95-106.

ROBINSON, W.R. y NÍAZ, M. (1991). Performance based on instruction by lecture or by interaction and its relationship to cognitive variables. International Journal of Science Education, 13(2), pp. 203-215.

ROTH, W.M. y LUCAS, K.B. (1997). From «Truth» to «Invented Reality»: A Discourse Analysis of High School Physics Students' Talk about Scientific Knowledge. Journal of Research in Science Teaching, 34(2), pp. 145-179.

ROTH, W.M. y ROYCHONDHURY, A. (1994). Students' Epistemologies and Views about Knowing and Learning. Journal of Research in Science Teaching, 31(1), pp. 5-30.

RUBA, P.A. y HARKNERSS, W.L. (1993). Examination of pre-service and in-service secondary science teachers' beliefs about Science/Technology/Society interactions. Science Education, 77(4), pp. 407-431.

RUGGIERI, R. TARSITANI, C. y VICENTINI, M. (1993). The images of science of teachers in Latin countries. International Journal of Science Education, 15(4), pp. 383393.

SANMARTÍ, N. y TARÍN, R. (1999). Valores y actitudes: ¿Se puede aprender ciencias sin ellos? Alambique, 22, pp. 55-65.

SELLEY, N.J. (1989). The philosophy of school science. Interchange, 20(2), pp. 24-32.

SOLOMON, J. (1987). Social influences on the construction of pupils' understanding of science. Studies in Science Education, 14, pp. 63-82.

SOLOMON, J. (1991). Teaching about the nature of science in the British National Curriculum. Science Education, 75(1), pp. 95-103.
SOLOMON, J., DUVEEN, J. y SCOTT, L. (1994). Pupils' images of scientific epistemology. International Journal of Science Education, 16(3), pp. 361-373.

SPECTOR, B., STRONG, P. y LA PORTA, T. (1998). Teaching the nature of science as an element of science, technology and society, en McComas, W.F. (ed.). The nature of science in science education. Rationales and strategies, pp. 267-276. Netherlands: Kluwer Academic Publishers.

SPERANDEO-MINEO, R. M. (1999). Epistemological beliefs of physics teachers about the nature of science and science models, en Proceedings Second International Conference of the European Science Education Research Association (ESERA). Research in Science Education: past, present and future, pp. 250-253.

STINNER, A. (1992). Science textbooks and science teaching: from logic to evidence. Science Education, 76(1), pp. 1-16.

SUTTON, C. (1998). New perspectives on language in science. en Fraser, B.J. y Tobin, K.G. (eds.). International Handbook of Science Education, 27-38. Great Britain : Kluwer Academic Publishers.

TEODORO, S. y NARDI, R. (2000). A História da Ciência e as concepções alternativas de estudantes como subsídios para o planejamento de um curso sobre atracção gravitacional. Cadernos de Textos, VEscola de Verão, pp. 278-280. Brasil: UNESP.

THOMAZ, M.F., CRUZ, M.N., MARTINS, I.P. y CACHAPUZ, A.F. (1996). Concepciones de futuros profesores del primer ciclo de primaria sobre la naturaleza de la ciencia: Contribuciones de la formación inicial. Enseñanza de las Ciencias, 14(3), pp. 315-322.

TOBIN, K.G., TIPPINS, D.J., y GALLARD, A.J. (1994). Research on Instructional Strategies for Teaching Science, en Gabel, D.L. (ed.). Handbook of Research on Science Teaching and Learning. Nueva York: National Science Teachers Association.

TOBIN, K.G., TIPPINS, D.J. y HOOK, K. (1994). Referents for changing a science curriculum: A case study of one teacher's change in beliefs. Science \& Education, 3, pp. 245264.

TRAVER y RIVES, M.J. (1996). «La història de les ciències en l'ensenyament de la física i la química». Tesis doctoral. Departament de Didàctica de les Ciències Experimentals. Universidad de Valencia.

WHEATLEY G.H. (1991). Constructivist Perspectives on Science and Mathematics Learning. Science Education, 75 (1), pp. 9-21.

YERRICK, R.K., PEDERSEN, J.E. y ARNASON, J. (1998). «We're just spectators»: A case study of science teaching, epistemology and classroom management. Science Education, 82(6), pp. 619-648. 